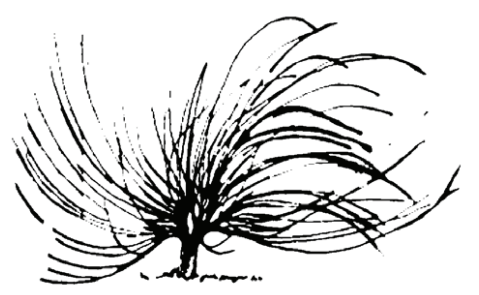

\title{
Propuesta metodológica en la enseñanza de la Física para desarrollar las bases matemáticas de los y las estudiantes de décimo año en un Colegio de Heredia
}

\author{
Evelyn Piedra Pereira ${ }^{1}$ \\ Universidad Nacional de Costa Rica \\ Cartago, Costa Rica \\ evelynpiedra8@hotmail.com \\ Karen Rojas Marchena ${ }^{2}$ \\ Universidad Nacional de Costa Rica \\ San José, Costa Rica \\ Karen89_rm@yahoo.com
}

\begin{abstract}
Resumen
La enseñanza de la Física en Costa Rica presenta una serie de problemas, los cuales ocasionan que los y las estudiantes pierdan el interés por esta ciencia, uno de ellos es las malas bases matemáticas que presentan los alumnos a nivel de decimo año, además no realizan un razonamiento físico y les falta el análisis en la resolución de los problemas. Ante esta necesidad, dicha investigación trata de diseñar una propuesta metodológica para mejorar el proceso de enseñanza-aprendizaje y conocer cuál es la actitud de los discentes cuando están en una lección de Física; asimismo lograr un desarrollo de las bases matemáticas de los y las estudiantes.
\end{abstract}

Recibido: 30 de noviembre de 2011 - Aprobado: 12 de marzo de 2012

1 Bachiller en Enseñanza de las Ciencias Naturales en la Universidad Nacional, Costa Rica y en la Universidad Estatal a Distancia (UNED) su Diplomado en Educación General Básica.

2 Bachiller en Enseñanza de las Ciencias Naturales en la Universidad Nacional, Costa Rica. 
Palabras clave: enseñanza de la Física, bases matemáticas, aprendizaje significativo, zonas de desarrollo próximo.

\begin{abstract}
Teaching physics in Costa Rica has a number of problems which causes the students to lose interest in this science; one of them is students' poor mathematical foundations at tenth grade, as well as there is no physical reasoning and there is a lack of analysis during solving problems. Against this requirement, this research is to design a proposal to improve the teaching-learning process and to know the attitude of the students during a physics lesson, likewise, achieve a development of the mathematical foundations of students.
\end{abstract}

Keywords: teaching physics, mathematical foundations, meaningful learning, zones of proximal development.

\title{
Introducción
}

n el sistema educativo costarricense se presentan una serie de
problemas en cuanto al proceso de enseñanza-aprendizaje en la
educación diversificada, debido a que existen docentes que no logran desarrollar las lecciones de una manera agradable y atractiva para los discentes, en especial, las de física, además la falta de interés por parte de los estudiantes en el área científica es deficiente porque, según Pozo y Gómez (1998) "los alumnos cada vez aprenden menos y se interesan menos de lo que aprenden" (p. 18).

Por esta razón, los estudiantes presentan poca motivación durante el desarrollo de las clases de física, porque coexiste una relación directa con las matemáticas, ya que en el área de las ciencias naturales la resolución de problemas es un factor necesario para su comprensión debido a que tienen que aplicar los conocimientos previos lógico-matemático en la resolución de éstos, lo que provoca un rechazo hacia la Física y dando como resultado un bajo rendimiento académico.

Dicha investigación se realizó en un colegio de Heredia, en la sección 10-2, en la clase de física, la cual se encontraba conformada por treinta estudiantes, que provenían de instituciones privadas y públicas. Este artículo, tiene como fin dar una perspectiva de lo que está 
aconteciendo en la enseñanza de la física en secundaria pública, ya que algunos estudiantes presentan problemas de aprendizaje en esta ciencia, debido generalmente a la falta de conocimientos previos lógico-matemático que son necesarios, para lograr la asimilación de los términos físicos, desarrollo y análisis de problemas. Además, es importante que el docente conozca el nivel matemático que poseen sus alumnos, con el objetivo de explicar los contenidos de una manera adecuada, en la cual desarrolle un aprendizaje significativo para todos los estudiantes y logren confrontar dicho problema con ayuda del profesor, compañeros e institución.

El impacto que esta situación tiene en la realidad, puede ocasionar que los alumnos no logren desarrollar la capacidad de resolver problemas que involucren las Matemáticas y la Física, ocasionando que estos no cumplan con sus objetivos hasta el punto de perder las materias. Desde la didáctica crítica se puede analizar que este problema provoca que los estudiantes deserten del sistema educativo, lo cual viene aumentar el índice de personas que no logran tener su título de bachillerato, además, no alcancen tener una educación superior, dando como resultado un pueblo sin educación que deja que el gobierno tome las decisiones por ellos. Por esta razón, es de suma importancia que los docentes puedan ayudar a los discentes a superar esos obstáculos y que no abandonen su educación. Dicho contexto se debe analizar no solo desde el punto de vista de la enseñanza de la Física, sino de todas las demás áreas del saber, ya que muestra lo que viven todos los días los estudiantes y profesores en el sistema educativo nacional.

\section{- Antecedentes}

En Costa Rica se han desarrollado estudios sobre la enseñanza de la física, en la "Influencia de las técnicas didácticas en la motivación hacia las clases de Física" (Hidalgo y Miranda, 2007) en la que proponen nuevas estrategias tales como: laboratorios y lecturas, además se encontró un estudio sobre "Factores que influyen en estudiantes de undécimo año para que no seleccionen Física como materia a presentar en las Pruebas Nacionales de Bachillerato" (Calvo y Espinoza, 2005), por otro lado, existen otros trabajos sobre la "Aptitud ante la física y las técnicas didácticas" (García, 2001).

Sin embargo, no se hallan investigaciones que establezcan una relación estrecha con las bases matemáticas en la resolución de 
problemas de Física, determinando que es un factor significativo para que los estudiantes obtengan un bajo rendimiento en dicha asignatura.

Arias y Jiménez (2004) analizaron las creencias relacionadas con la física: Física como ciencia, al profesor de Física y su papel en el proceso didáctico, los cuales presentan relación con la física en el ciclo diversificado. Como resultado obtuvieron que los estudiantes tiene una mala idea de lo que se refiere a la física, porque les cuesta aplicar teoría o los fenómenos físicos en la resolución de los problemas, diciendo que la matemática aplica solo fórmulas, lo cual genera un conflicto entre la física y la matemática, algunos estudiantes se les hace más fácil la matemáticas y presenta problemas en física. Por último, Rojas (2006), crea las “Zonas de Desarrollo Próximo como estrategias en enseñanza-aprendizaje constructivista en la resolución de problemas lógico-matemático en una clase de física", el cual tiene como resultado que los estudiantes asumen roles diferentes en el trabajo en equipo, pero hay grupos que no logran establecerlo bien. Además, que el carácter de heterogeneidad en los equipos de trabajo potencia el desarrollo de Zonas de Desarrollo Próximo, es decir, los alumnos obtienen mayores logros cuando comparten la tarea, por lo que favorece el aprendizaje a nivel cognitivo.

\section{Problema}

¿De qué forma una propuesta metodológica en la enseñanza de la Física mejoraría las bases matemáticas que presentan los y las estudiantes en la sección 10-2?

\section{Objetivo general}

Analizar la metodología implementada en la enseñanza de la Física para desarrollar las bases matemáticas de los y las estudiantes de décimo año.

\section{Objetivos específicos}

a) Reconocer los problemas matemáticos que presentan los y las estudiantes en un colegio en Heredia, en la enseñanza de la Física en la sección 10-2.

b) Identificar las estrategias metodológicas utilizadas por el docente en las lecciones de Física en la sección 10-2.

c) Caracterizar la actitud de los estudiantes en el área de la Física. 
d) Diseñar una propuesta metodológica, para desarrollar las bases matemáticas de los estudiantes de décimo año en la enseñanza de la Física.

\section{Referente teórico}

\section{La Física en la educación secundaria}

El propósito de la enseñanza es preparar a los estudiantes hacia un desarrollo integral, lo cual conlleva la incorporación en la sociedad, a través de las distintas materias que forma el currículum escolar (Comisión de Educación ANQUE, 2005). Dichas materias deben ir destinadas a crear en los discentes conocimientos y a desarrollar actitudes y hábitos que respondan a una adecuada calidad de vida en sociedad.

La enseñanza de la Física en secundaria es de suma importancia ya que forma parte de lo que se conoce como ciencias básicas porque es la base para otras ciencias e ingenierías (González, 2005). Además el conocimiento de la Física, junto con el resto de las materias presentes en el currículum, resulta imprescindible para comprender de una mejor manera el desarrollo social, económico y tecnológico en el que nos encontramos; así como para poder participar con criterios propios ante algunos de los grandes problemas que la sociedad tiene en la actualidad (Comisión de Educación ANQUE, 2005).

En Costa Rica, un gran número de estudiantes no logran comprender la Física, lo cual se atribuye a que muchos de esos alumnos no tienen o les hace falta ciertas bases lógico matemáticas, para comprender y asimilar los contenidos físicos. Por otro lado, muchos de los alumnos que se encuentran en las pruebas de Bachillerato no escogen realizar la prueba de Física ya que para poder resolver problemas físicos se requiere de un alto nivel de desarrollo mental, conocimiento, comprensión, aplicación y análisis de información (Rojas, 2006).

\section{Problemas en la enseñanza de la Física}

De acuerdo con Pozo y Gómez (1998), una de las dificultades principales de los estudiantes van a estar denominadas por la forma en que ven al mundo, mientras que al final lo estará por la forma en que no lo ve (p. 209). Las dificultades durante el desarrollo de la Física en secundaria se presentan en la tabla 1 . 
Tabla 1. Dificultades de los estudiantes en aprendizaje de la Física

\begin{tabular}{|c|c|c|c|}
\hline & Se estudian & Mediante & Dificultades generadas por \\
\hline 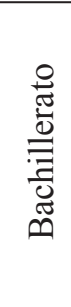 & $\begin{array}{l}\text { Fenómenos próximos al } \\
\text { alumno. } \\
\text { Fenómenos más allá de } \\
\text { lo observable }\end{array}$ & $\begin{array}{l}\text { Profundización } \\
\text { en conceptos y } \\
\text { procedimientos de } \\
\text { trabajo. } \\
\text { Alta carga del estudio } \\
\text { cuantitativo de los } \\
\text { fenómenos. }\end{array}$ & $\begin{array}{l}\text { La forma en que el } \\
\text { alumno ve el mundo } \\
\text { Necesidad de representar } \\
\text { lo no observable } \\
\text { Cálculos matemáticos y } \\
\text { resolución de problemas } \\
\text { cuantitativos. }\end{array}$ \\
\hline
\end{tabular}

Adaptado de: Pozo y Gómez (1998)

Los alumnos no encuentran dificultades conceptuales, sino que se presentan en el uso de las estrategias de razonamiento (lógico - matemático) y en la solución de problemas que son propios de la Física, por su parte, según Pozo y Gómez, (1998), citados por Hidalgo y Miranda (2007), es una ciencia teórico-práctica que el aprendizaje requiere de conocimientos fundamentales en matemáticas debido a que los ejercicios tradicionales y problemas numéricos implican manipulación de datos numéricos y es aquí donde se percibe la dificultad. (p.12).

Desde esta perspectiva, los problemas matemáticos se pueden incorporar a los problemas físicos y que el estudiante centre su atención en el aprendizaje de técnicas y algoritmos de cálculo olvidando el contenido científico (Pozo y Gómez, 1998).

Todo esto refleja la manera en la que la enseñanza de la Física ha tenido problemas que están entrelazos con las bases matemáticas, ocasionando que cambie la forma de aprender Física en el contexto natural.

\section{Rol del docente en la enseñanza de la Física}

Los profesores son una parte importante en la enseñanza de la física, porque ellos (as) deben desarrollar en los estudiantes una cultura sobre lo que es ciencia, en este caso la Física; por su parte, Briceño et al. (2005) consideran que los profesores de Física, “... deben preparar a los estudiantes con un grado de actualización que le permita vivir acorde con su época, es necesario fomentar en ellos el espíritu crítico y valorativo ante la realidad que se les presenta...." (p.2).

Para un profesor de secundaria es necesario conocer los problemas que se enfrentan en la enseñanza de la Física, para lograr resultados 
óptimos en el desarrollo de las lecciones y hacer más gratificante su labor. Por último, el docente debe saber cada una de las dificultades que presentan los estudiantes en la materia, en cuanto a los problemas de aprendizaje o en el área de las matemáticas.

\section{Relación de la Física y las Matemáticas \\ Sandonís, (s.f) propone lo siguiente:}

Las matemáticas son por lo tanto el lenguaje que utiliza la Física para expresar el orden en la naturaleza, para expresar la relación entre las diversas magnitudes físicas que podemos medir al estudiar los fenómenos naturales. Pero, que las matemáticas sean esenciales en la Física no debe confundirse con la idea de que "la Física es pura Matemática". Las diferentes letras de una fórmula física no se corresponden con algo abstracto, puramente matemático, sino que representan conceptos físicos, aspectos ligados a la realidad que nos rodea. Este aspecto debe ser considerado siempre que se hagan cálculos matemáticos. El resultado de todos esos cálculos matemáticos tiene que tener sentido físico. (p.2).

La Física se considera una de las disciplinas que forma parte de las ciencias de la naturaleza, que como objetivo tiene el estudio del mundo y de sus fenómenos (Pozo y Gómez, 1998). Por lo que, los estudiantes deben hacer un análisis del fenómeno físico que se le presenta en el problema o ejercicio y luego hacer el razonamiento lógico- matemático. La relación de la Física con la Matemática en secundaria se presenta en la siguiente fórmula.

\section{Física $=$ Razonamiento físico + matemáticas}

Adaptado de: Sandonís (s.f)

\section{Estrategias metodológicas en la enseñanza de la Física: Crea- ción de Zonas de Conocimiento Próximo}

La física constituye una herramienta muy útil para la comprensiónde una gran parte de la ciencia y la tecnología actual, así como diferentes fenómenos sociales, por esta razón 
forma parte del currículum en la enseñanza secundaria (González, 2011), ya que según Vigotsky en su teoría de "Zonas de Desarrollo Próximo", el individuo se constituye de un aislamiento, más bien en una interacción, donde influyen mediadores que guían al individuo a desarrollar sus capacidades cognitivas, además, ZDP es el lugar donde, gracias a los soportes y a la ayuda de otros, pueden desencadenar el proceso de construcción, modificación, enriquecimiento y diversificación de los esquemas de conocimiento que define el aprendizaje escolar (Rojas, 2006).

Por esta razón, es de suma importancia implementar distintas estrategias metodológicas que desarrollen en los discentes un aprendizaje significativo. Bernaza, Corral y De La Peña (2006), proponen lo siguiente:

- Prácticas de laboratorio como base en el "aprendizaje por descubrimiento".

- La transmisión-recepción de conocimientos como garantía de un aprendizaje significativo.

- Utilización de las computadoras en la enseñanza de la física.

- Propuestas constructivistas como eje de transformación de la enseñanza de las ciencias.

Por otro lado, se debe incentivar a los estudiantes a la resolución de problemas en que los estudiantes, no solo los resuelvan sino que deban emplear sus conocimientos de forma crítica y asimismo, desarrollar el trabajo cooperativo.

\section{Aprendizaje significativo}

González (2011) establece que:

Un aprendizaje significativo es cuando los contenidos son relacionados de modo no arbitrario y sustancial; por esta relación sustancial y no arbitraria se debe entender que "las ideas se relacional con algún aspecto existente específicamente relevante de la estructura cognoscitiva del alumno, como una imagen, un símbolo ya significativo, un concepto o una proposición (p.2). 
En el proceso de enseñanza aprendizaje se dan dos tipos de aprendizaje los cuales son: el memorístico o mecánico y el significativo. Lamentablemente, el aprendizaje memorístico es el que prevalece en su mayoría en los salones de clase costarricense, un ejemplo de ello, sería el simple hecho de memorizarse los procedimientos de algún problema físico, dejando de lado el análisis necesario para llegar a la respuesta.

El aprendizaje mecánico, al no estar relacionado con los conocimientos anteriores que poseen los discentes, la retención es mucho menor. Al mismo tiempo, resulta casi imposible utilizar dichos conocimientos en la vida cotidiana (González, 2011), también en el caso de la física el estudiante presentaría un gran problema, porque dicha materia se encuentra estrechamente relacionada con otros términos tanto físicos como lógico-matemáticos.

Para obtener un verdadero aprendizaje significativo en los alumnos, se debe partir de los conocimientos previos que poseen. Por esta razón, el docente debe brindar a los estudiantes la ayuda necesaria para afrontar los problemas que tengan en su proceso de enseñanza-aprendizaje.

\section{Metodología}

El proyecto de investigación se encierra en el paradigma naturalista-humanista, según Barrantes (1999), "se centra en el estudio de los significados de las acciones humanas y de la vida social" (p.60), cuyo fin es comprender e interpretar la realidad, los significados de las personas, percepciones, interacciones y acciones" (p.61), además este paradigma se sitúa bajo el enfoque cualitativo. Según Hernández, Fernández y Baptista (2006), "el enfoque cualitativo, es aquel tipo de estudio que busca comprender un fenómeno de estudio en un ambiente inusual (cómo se comporta, actúa, piensa, etc)" (p.12). Además, estos autores añaden que los investigadores están directamente involucrados con las personas que se estudian y con sus experiencias personales.

A partir de los objetivos planteados y la metodología de la investigación se trata de comprender el comportamiento y la actitud que presentan los estudiantes en relación con las bases matemáticas en la enseñanza de la física; por lo tanto, se pretende dar una propuesta metodológica. 
Esta investigación es de tipo investigación-acción, según Restrepo (s.f):

Concibió este tipo de investigación como la emprendida por personas, grupos comunidades que llevan a cabo una actividad colectiva en bien de todos, consistente en una práctica reflexiva social en la que interactúan la teoría y la práctica con miras a establecer cambios apropiados en la situación estudiada y en la que no hay distinción entre lo que se investiga, quién investiga y el proceso de investigación (p.2).

Con esta investigación se pretende diseñar una propuesta metodológica, para desarrollar las bases matemáticas en los alumnos.

\section{- Participantes: profesor colaborador y estudiantes del grupo en estudio}

La investigación se realizó con una población de treinta estudiantes y el profesor colaborador. El grupo es un décimo año, el cual fue estudiado en las lecciones de Física, en el Colegio Humanístico Costarricense de la Dirección Regional de Heredia.

\section{- $\quad$ Recolección de la información}

El grupo de estudio fue escogido intencionalmente por las investigadoras con el fin de identificar alguna problemática en la sección, asimismo, se hizo la negociación con el docente colaborador para así poder realizar el trabajo, en el cual se desarrolló una entrevista informal para obtener una visión de los posibles problemas que presentaba el grupo.

Para la obtención de la información de la sección en estudio se utilizaron, las siguientes técnicas: tres observaciones, cuestionario al grupo y ntrevista formal al profesor.

\section{- Observación}

Según Santamaría (2006): "La observación es un proceso, que demanda una buena dosis de interés y requiere de un registro sistemático de datos" (p. 62).

Se dieron tres observaciones, la primera se desarrolló durante la aplicación del primer examen parcial de Física, posteriormente, se realizaron dos observaciones semiestructural-no participativa, para 
reconocer la perspectiva que presentan los discentes en relación con la Física -Matemática, profesor-estudiante y estudiante-estudiante.

\section{- Cuestionario}

Con este instrumento se trató de conocer la opinión de los alumnos acerca de sus lecciones de Física y cómo les gustaría que las impartieran, si presentaban algunas dificultades Matemáticas y en Física, a partir de preguntas abiertas y cerradas.

Para Fromm y Ramos (2009) consideran un cuestionario consideran como:

Un inventario de preguntas dirigidas a la recolección de ciertos datos relacionados con la variable que se pretende medir. Así, no contienen cualquier pregunta. El cuestionario posee dos tipos de preguntas: abiertas o cerradas.

Las preguntas cerradas consisten en preguntas con respuestas previamente delimitadas por el investigador... Las preguntas abiertas, al contrario, no cuentan con respuestas predeterminadas. Por tanto, no delimitan de antemano las alternativas de respuestas. (p. 65).

\section{- $\quad$ Entrevista al docente}

La eentrevista es una técnica para obtener información mediante una conversación profesional con una o varias personas. Este proceso implica un diálogo franco, en donde la comunicación entre los docentes-investigadores y los participantes ocurre de manera directa (Hernández et al., 2006).

Al final de la recolección de datos, se realizó una entrevista formal al docente, con la intención de que el docente colaborador a partir de su experiencia profesional en la enseñanza de la Física suministre su punto de vista acerca del problema planteado por las investigadoras.

\section{Análisis y resultados}

Este apartado reúne los resultados obtenidos del trabajo de campo que se desarrolló con el objetivo de conocer la metodología implementada en la enseñanza de la Física para desarrollar las bases matemáticas de los y las estudiantes de décimo año. 
A continuación se presenta el análisis de los datos obtenidos, mediante la aplicación respectiva de observaciones al grupo, entrevista al profesor colaborador y cuestionario para todo el grupo.

\section{Matriz $\mathbf{N}^{\circ} 1$ \\ Principales dificultades y actitudes que presentan los estudiantes en la enseñanza de la Física en un colegio de Heredia}

\begin{tabular}{|l|l|}
\hline \multicolumn{1}{|c|}{ Pregunta } & \multicolumn{1}{|c|}{ Respuestas } \\
\hline $\begin{array}{l}\text { 1. ¿Cuáles son las principales difi- } \\
\text { cultades que presentan los estudi- } \\
\text { antes en dicha materia? }\end{array}$ & $\begin{array}{l}\text { Tienen deficiencias sobre el procedimientos } \\
\text { matemáticos, como despeje de fórmulas, proced- } \\
\text { imientos algebraicos, trigonometría y también } \\
\text { mala disposición a la materia }\end{array}$ \\
\hline $\begin{array}{l}2 \text { ¿Cómo ha podido solucionar estos } \\
\text { problemas los años anteriores? }\end{array}$ & $\begin{array}{l}\text { En la medida de lo posible se trata de explicar } \\
\text { un poco los procedimientos que se necesitan en } \\
\text { clase y se trabaja mucho con motivación. }\end{array}$ \\
\hline $\begin{array}{l}\text { 2. Usted como profesor, ¿Cómo } \\
\text { considera que es la actitud de los } \\
\text { estudiantes en las clases de Física? }\end{array}$ & $\begin{array}{l}\text { Regular, muchos llegan con la idea de que es una } \\
\text { pateria difícil y por eso no se esfuerzan y otros } \\
\text { pueden salir adelante. }\end{array}$ \\
\hline $\begin{array}{l}\text { 4. Cómo considera usted que los } \\
\text { estudiantes catalogan las clases de } \\
\text { Física? ¿Por qué? }\end{array}$ & $\begin{array}{l}\text { Muchos llegan con el concepto que es difícil, } \\
\text { peor es porque muchos profesores hacen que sea } \\
\text { complicada para ellos (as). }\end{array}$ \\
\hline $\begin{array}{l}\text { 5. Considera usted que las bases } \\
\text { matemáticas son un aspecto impor- } \\
\text { tante en la enseñanza de la Física. } \\
\text { ¿Por qué? }\end{array}$ & $\begin{array}{l}\text { Sí, por el tipo de habilidades que desarrolla el es- } \\
\text { tudiante, esto hace que su capacidad de análisis e } \\
\text { interpretación sea mejor. }\end{array}$ \\
\hline
\end{tabular}

Fuente: entrevista al profesor colaborador.

Se puede observar que los principales problemas que presentan los estudiantes de esta sección (Ver matriz 1) se debe a los principios matemáticos esenciales para comprender Física de la mejor manera. Se les dificulta realizar despeje de fórmulas, procedimientos algebraicos, trigonometría y también mala disposición la hacia la materia, que en muchos casos se debe a que los docentes que tuvieron en años anteriores no les ayudaron a desarrollar de manera correcta los contenidos o que crearon en ellos la idea de que Física es una materia "difícil". Cuando en realidad no es así, debido a que, no solo se necesita un conocimiento matemático, sino un razonamiento del fenómeno que ocurre, 
(se debe recalcar que en esta institución ingresan discentes tanto de colegios públicos y privados, lo que según el profesor colaborador, ésta podría ser una causa de deficiencia que presentan los estudiantes en la Matemática. (Ver matriz 2).

\section{Matriz $\mathbf{N}^{\circ} 2$}

\section{Comparación entre los estudiantes que les gusta y} los que no les gusta las Matemáticas

\begin{tabular}{|c|c|c|}
\hline \multicolumn{3}{|c|}{ Pregunta 1: ¿Le gusta la matemáticas? } \\
\hline Estudiantes & $\begin{array}{c}\text { Respuesta de } \\
\text { los estudiantes }\end{array}$ & Justificación de la respuesta \\
\hline & Sí o No & \\
\hline $\begin{array}{l}1,2,3,6,7,8, \\
9,10,11,12, \\
13,14,15,16, \\
17,18,19,20 \\
21,22,23,27\end{array}$ & Sí & $\begin{array}{l}\text { 1. Me gustan los números y en sí, me gusta } \\
\text { todo lo relacionado con ellos, además se me } \\
\text { hacen muy fáciles Me gusta resolver las op- } \\
\text { eraciones, me gusta especialmente el álgebra } \\
\text { en Matemáticas. }\end{array}$ \\
\hline $4,5,24,25,26$ & No & $\begin{array}{l}\text { 1. Se tiene que utilizar la mente cuando no uso } \\
\text { la calculadora. } \\
\text { 2. Es aburrida. } \\
\text { 3. No es uno de los campos donde tenga más } \\
\text { talento sinceramente, no es que no me gusten } \\
\text { es que me cuestan. } \\
\text { 4. Porque no soy muy buena para resolver la } \\
\text { matemática. } \\
\text { 5. Me cuesta entenderlo completamente y } \\
\text { siempre fallo en errores muy obvios. }\end{array}$ \\
\hline
\end{tabular}

Fuente: cuestionario relación de la física y matemáticas. 
A pesar de que algunos estudiantes presentan dificultades en matemáticas, son muy pocos los que no les gusta, además, comparando matriz 2 y 3 se puede rescatar que algunos estudiantes no les gusta la materia; sin embargo no presentan problemas para comprenderla. Por otro lado, los estudiantes que no les agradan las matemáticas, puede ser que no han desarrollado un razonamiento lógico-matemático adecuado para su comprensión.

\section{Matriz $\mathbf{N}^{\circ} 3$}

\section{Contenidos de Matemáticas que presentan una mayor dificultad los (as) estudiantes}

\begin{tabular}{|l|l|}
\hline \multicolumn{2}{|c|}{ Pregunta 2: ¿En cuáles temas presenta mayor dificultad de comprensión? } \\
\hline \multicolumn{1}{|c|}{ Actores } & \multicolumn{1}{c|}{ Respuesta } \\
\hline Estudiante 4 & Todos los entiendo solo necesito concentrarme y poner atención. \\
\hline Estudiante 5 & $\begin{array}{l}\text { En realidad yo comprendo pero simplemente no me gustan los } \\
\text { números. }\end{array}$ \\
\hline Estudiante 6 & $\begin{array}{l}\text { Temas que hay que poner mucha atención en detalles de algunas } \\
\text { factorizaciones. }\end{array}$ \\
\hline Estudiante 7 & Álgebra. \\
\hline Estudiante 9 & Álgebra y geometría. \\
\hline Estudiante 24 & $\begin{array}{l}\text { Álgebra, este es el tema que más me cuesta, luego la geometría, } \\
\text { pero no tanto como álgebra. }\end{array}$ \\
\hline Estudiante 25 & En la mayoría de temas que tiene matemáticas. \\
\hline Estudiante 26 & En matemáticas, física-mate. \\
\hline
\end{tabular}

Fuente: cuestionario relación de la física y matemáticas. 


\section{Matriz $\mathrm{N}^{\circ} 4$}

\section{Percepción de los estudiantes acerca de la lección de Física impartida por su docente}

\begin{tabular}{|c|c|c|}
\hline \multicolumn{3}{|c|}{ Pregunta 7: ¿Le gusta la lección de Física impartida por el docente? } \\
\hline & $\begin{array}{c}\text { Respuesta de } \\
\text { los estudiantes }\end{array}$ & \\
\hline Actores & Sí o No & Justificación de la respuesta \\
\hline $\begin{array}{l}1,2,3,6,7, \\
8,9,10,11, \\
12,13,14,15, \\
16,17,18,19, \\
20,21,22,23, \\
24,25,26,27\end{array}$ & Sí & $\begin{array}{l}\text { 1. El profesor logra mantener al grupo atento y a } \\
\text { veces para lograrlo bromea muy poco pero lo } \\
\text { hace para mantenernos atentos. } \\
\text { 2. Explica muy bien y hace la clase divertida. } \\
\text { Tiene paciencia y es comprensible. } \\
\text { 3. Explica muy claramente, es ordenado, la práctica } \\
\text { va de acuerdo con como hemos visto la materia. } \\
\text { 4. Atiende dudas con paciencia y es una lección } \\
\text { muy dinámica e interesante. } \\
\text { 5. La mayoría de veces, en mi cole anterior no } \\
\text { entendía lo que me explicaban, aquí sí. Y sus } \\
\text { explicaciones son buenas. }\end{array}$ \\
\hline
\end{tabular}

Fuente: cuestionario relación de la física y matemáticas.

En lo que respecta al docente, los alumnos consideran que las lecciones y estrategias empleadas por el profesor son las más adecuadas, porque todos mencionaban que comprenden todo lo que éste les enseña, también comentaban que la forma en que él imparte la clase es ordenada, amena y divertida, por supuesto, que desarrolla los contenidos de forma clara (Ver matriz 4), las estrategias metodológicas empleadas por el docente colaborador, son sencillas y claras, lo cual facilita la comprensión de los contenidos dados, conjuntamente, se puede rescatar que el profesor trabaja sus lecciones a partir de la motivación, lo que según Hidalgo (2007), aquellos individuos que se encuentren motivados presentaran una disposición y deseo por las tareas que realice, esto resulta de suma importancia en la educación formal (Ver matriz 5 y 6); además el docente es paciente y respetuoso con las dudas que presentan los estudiantes en clase. 
Matriz $\mathbf{N}^{\circ} \mathbf{5}$

Comparación entre los estudiantes que les gusta y los que no la Física

\begin{tabular}{|c|c|c|}
\hline \multicolumn{3}{|c|}{ Pregunta 4: ¿Le gusta la física? } \\
\hline Actores & \begin{tabular}{|c|} 
Respuesta de \\
los estudiantes
\end{tabular} & \\
\hline & Sí o No & Justificación de la respuesta \\
\hline $\begin{array}{l}1,2,3,6,7, \\
8,9,10,11, \\
12,13,14,15, \\
16,17,18,19, \\
20,21,22,23, \\
24,25,26\end{array}$ & Sí & 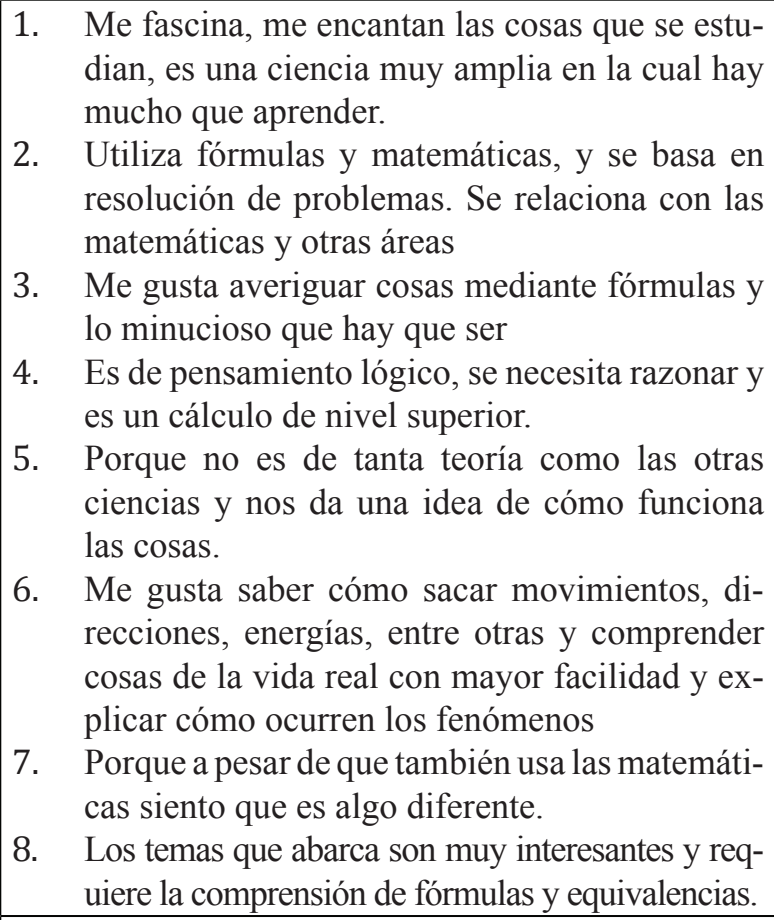 \\
\hline Estudiante 27 & No & $\begin{array}{l}\text { No es que no me guste, simplemente es que me } \\
\text { cuesta un poco. }\end{array}$ \\
\hline
\end{tabular}

Fuente: cuestionario relación de la física y matemáticas. 


\begin{tabular}{|l|l|}
\multicolumn{1}{l}{$\begin{array}{l}\text { Mvatriz No } 6 \\
\text { realizadas durante dos semanas en las lecciones } \\
\text { de Física, en un colegio de Heredia }\end{array}$} \\
\begin{tabular}{|l|l|}
\hline \multicolumn{1}{|c|}{ Actores } & \multicolumn{1}{c|}{ Observaciones } \\
\hline Profesor & $\begin{array}{l}\text { Motiva a los estudiantes en las explicaciones teóricas y prácticas } \\
\text { de los temas. } \\
\text { El profesor desarrolla clases magistrales y comienza la materia me- } \\
\text { diante un ejemplo. Dispone de un resumen de la materia y prácticas } \\
\text { para resolver en la pizarra. } \\
\text { El docente aclara dudas a los y las estudiantes de manera correcta. } \\
\text { Atiende de forma individual las dudas que presentan. }\end{array}$ \\
\hline Estudiantes & $\begin{array}{l}\text { Los estudiantes muestran interés y están atentos a las explicaciones } \\
\text { del profesor. Realizan preguntas durante las explicaciones, es decir, } \\
\text { la participación es excelente. Mucho presentan problemas para } \\
\text { comprender la materia. } \\
\text { Hay estudiantes que no socializan y realizan las prácticas } \\
\text { individualmente. }\end{array}$ \\
\hline $\begin{array}{l}\text { Estudiantes- } \\
\text { Profesor }\end{array}$ & $\begin{array}{l}\text { El profesor toma en cuenta los problemas que presenta los estudi- } \\
\text { antes en matemáticas (trigonometría), trata de dar una breve ex- } \\
\text { plicación del tema sin desviarse de los contenidos de la materia. } \\
\text { Además es respetuoso. } \\
\text { No saben realizar despejes en el temas de física. } \\
\text { Habla con los estudiantes durante el desarrollo de las prácticas. Estos } \\
\text { van hacia el docente para que les revise la prácticas y aclara dudas. }\end{array}$ \\
\hline Estudiante- \\
Estudiante
\end{tabular} $\begin{array}{l}\text { Se respetan y no critican a los compañeros (as) cuando presenta } \\
\text { dudas tanto en temas de física y matemáticas. } \\
\text { Trabajan de forma individual, parejas y grupos. Aplicados a la hora de } \\
\text { resolver las practicas de materia. Hay compañerismo y se ayudan entre } \\
\text { sí. Es un excelente grupo y les gusta, además que se motivan cuando } \\
\text { terminan el trabajo, sin embargo algunos no entendían la materia. }\end{array}$ \\
\hline
\end{tabular}

Fuente: elaboración propia a partir de observaciones durante dos semanas en las lecciones de física.

Las estrategias metodológicas preferidas por los estudiantes en la enseñanza de la Física son: demostraciones, laboratorios y talleres, las cuales son de suma importancia cognitiva, para percibir el fenómeno físico que ocurre, obviando un poco el razonamiento matemático y lograr un pensamiento científico creando una relación con su entorno. 


\section{Matriz $\mathbf{N}^{\circ} 7$ \\ Conocimiento de los estudiantes acerca relación entre Matemáticas y Física}

\begin{tabular}{|c|c|c|}
\hline \multicolumn{3}{|c|}{ Pregunta 7: ¿Cree que existe una relación entre Matemáticas y Física? } \\
\hline & $\begin{array}{c}\text { Respuesta de } \\
\text { los estudiantes }\end{array}$ & \\
\hline Actores & Sí o No & Justificación de la respuesta \\
\hline $\begin{array}{l}1,2,3,6,7, \\
8,9,10,11, \\
12,13,14,15, \\
16,17,18,19, \\
20,21,22,23, \\
24,25,26,27\end{array}$ & Sí & $\begin{array}{l}\text { 1. La Física ocupa de las matemáticas para ex- } \\
\text { plicar las cosas que la teoría no hace. } \\
\text { 2. La Física utiliza en gran parte números y fór- } \\
\text { mulas matemáticas } \\
\text { 3. La Física utiliza un lenguaje matemático, } \\
\text { formulas y leyes. } \\
\text { 4. Para ambas materias los procedimientos son } \\
\text { esenciales para la obtención de resultados. } \\
\text { 5. Los dos involucran procedimientos de análi- } \\
\text { sis y se resuelven problemas de manera pre- } \\
\text { cisa y exacta. } \\
\text { 6. Porque en Física se utilizan muchas fórmulas } \\
\text { y números (Los problemas que se plantean se } \\
\text { resuelven de manera práctica con fórmulas } \\
\text { matemáticas. } \\
\text { Un ejemplo Pitágoras }\end{array}$ \\
\hline
\end{tabular}

Fuente: cuestionario relación de la física y matemáticas.

La disposición que presentan los estudiantes hacia las lecciones de Física es un factor determinante para lograr un entendimiento de esta ciencia, según lo mencionado por el docente (Ver matriz 1). Los autores muestran una actitud regular porque muchos llegan con la idea de que es una materia difícil. Por eso no se esfuerzan y otros piensan que con poco estudio y poca práctica pueden salir adelante. A pesar de que tienen una idea errónea sobre la Física, la mayoría les gusta dicha materia. Además, a los que no les gusta, es porque la relacionan con matemáticas, según la matriz 7, ellos (as) tienen el conocimiento de que esta ciencia se encuentra asociada a los principios matemáticos elementales.

A partir de las observaciones realizadas por las investigadoras durante dos semanas en las lecciones de Física, se determinó que los estudiantes muestran interés y están atentos a las explicaciones del profesor, además realizan preguntas durante las explicaciones, es decir, la participación es excelente, sin embargo, éstos no socializan entre sí y realizan las prácticas individualmente, manteniendo el respeto hacia los 
compañeros que presentan problemas tanto matemáticos como físicos (Ver matriz 7). Esta metodología ejecutada por los estudiantes puede ser una forma que disminuya los problemas que presentan ya sea de contenidos físicos o matemáticos. Según Vigotsky en su teoría de "Zonas de Desarrollo Próximo”, el individuo se constituye de un aislamiento, más bien en una interacción, donde influyen mediadores que guían al individuo a desarrollar sus capacidades cognitivas, además una ZDP es el lugar donde, gracias a los soportes y a la ayuda de otros, pueden desencadenarse el proceso de construcción, modificación, enriquecimiento y diversificación de los esquemas de conocimiento que define el aprendizaje significativo.

\section{Propuesta}

Existe una diferencia entre aprender Física y Matemáticas ya que primero conseguimos un problema de Física, después lo expresamos en un problema matemático, entonces se resuelve el problema, haciendo una relación entre el fenómeno físico y los métodos matemáticos.

Por lo que consideramos crear una propuesta metodológica para desarrollar las bases matemáticas de los estudiantes de décimo año, sin embargo, la solución a este problema está en manos del docente, debido a que éste debe tener los elementos pedagógicos fundamentales en la metodología para resolver problemas que involucre Física y Matemáticas (De la Peña, sf).

Según los resultados y análisis de la investigación, se plantean algunas soluciones al problema:

1. Realizar una prueba diagnóstica, la cual tenga contenidos de matemáticas (como despeje de fórmulas, procedimientos algebraicos y trigonometría) a los y las estudiantes, para tener una percepción de los contenidos matemáticos que dominan los estudiantes de decimo año.

2. Taller de nivelación de las bases matemáticas, se impartirá después de obtener los resultados de los diagnósticos, para adquirir una visión de cuáles son los principales contenidos matemáticos que presentan dificultad los estudiantes.Para el desarrollo de estos talleres los profesores encargados, lo harán por medio de diferentes problemas físicos que involucre contenidos matemáticos, 
con ellos se pretende aclarar las dificultades que los dicentes tienen al respecto.

3. Taller de física en la vida, a partir de "Zonas de Desarrollo Próximo”, los estudiantes resolverán problemas físicos, los cuales permiten explorar los conocimientos previos que poseen los estudiantes sobre el fenómeno a estudiar. Por medio de interacciones (individual, pareja o tríos), así mismo explicar el problema propuesto por el docente a sus compañeros de manera creativa, para logra obtener aprendizajes significativos del orden conceptual, procedimental y actitudinal.

\section{Elaboración del manual}

Para lograr el desarrollo de los puntos anteriores, se propuso la idea de elaborar un manual que contuviera ciertos conceptos matemáticos y físicos, que tenían mayor dificultad de comprensión para los discentes. Además, que fueran útiles y necesarios para entender y así poder desenvolverse de una manera óptima, cuando tuvieran que resolver problemas de física (Taller de física para la vida). Dichos problemas serían pensados y elaborados a partir de las “Zonas de Desarrollo Próximo” con el fin de que los estudiantes establezcan relaciones de sus conocimientos previos y así desarrollen un aprendizaje significativo.

\section{Conclusiones}

- Se reconoció que los principales problemas matemáticos que presentan los y las estudiantes en un colegio de Heredia en la enseñanza de la Física en la sección 10-2 son el despeje de fórmulas, procedimientos algebraicos, trigonometría y también mala disposición a la materia, además muestran algunas dificultades conceptuales sobre matemática de años anteriores.

- Además, los alumnos establecen una relación directa entre la Matemática y la Física, por lo que piensan que es una materia meramente de carácter matemático, dejando de lado el análisis físico.

- Las estrategias metodológicas utilizadas por el docente en las lecciones de Física en la sección 10-2, son meramente magistrales; aunque se logra una asimilación adecuada de los contenidos enseñados. 
Propuesta metodológica en la enseñanza de la Física para desarrollar las bases matemáticas de los y las

- $\quad$ El docente desarrolla sus clases a partir de la motivación, lo cual es un factor que favorece el aprendizaje significativo de los estudiantes hacia la Física.

- La actitud de los estudiantes en el área de la Física, en su mayoría es buena; pero algunos piensan que es una ciencia de difícil comprensión.

\section{Referencias bibliográficas}

Arias, L. \& Jiménez, J. (2004). Cómo aprendemos física? Un análisis de las creencias de doce estudiantes del ciclo diversificado en el Liceo San Gabriel-La Salle. Costa Rica: Heredia. Universidad Nacional.

Barrantes, R. (1999). Investigación: Un camino al conocimiento. Un enfoque cualitativo y cuantitativo. San José, Costa Rica: Editorial Universidad Estatal a Distancia.

Bernaza, G, Corral, R \& De La Peña, C. (2006). Una propuesta didáctica para el aprendizaje de la Física. Universidad "José Marti”", Cuba. Recuperado de http:// www.rieoei.org/experiencias110.htm

Briceño, M., Díaz, J., Gutiérrez, G., Lobo, H., Rosario, J. \& Villarreal M. (2005). La enseñanza de la Física frente al nuevo milenio. Recuperado de http://www. saber.ula.ve/bitstream/123456789/16941/2/articulo1.pdf

Calvo, I. \& Espinoza, J. (2005). Factores que influyen en los estudiantes de undécimo año para que no seleccionen física como materia a presentar en las pruebas nacionales de bachillerato. Costa Rica: Heredia. Universidad Nacional.

Comisión de Educación ANQUE (Asociación Nacional de Químicos Españoles). (2005). La Enseñanza de la Física y la Química. Revista Eureka sobre Enseñanza y Divulgación de las Ciencias, Vol. 2, No 1, pp. 101-106. Recuperado de En http://www.apac-eureka.org/revista/Volumen2/Numero_2_1/Manifiesto-ANQUE.pdf

De la Peña, R. (s.f). Como enseñar a resolver problemas de física y matemáticas. Recuperado en http://www.galeon.com/metodologia/

González, A. (2011). La Física en 2005 y el Aprendizaje Significativo. Revista Iberoamericana de Educación. Recuperado de En http://www.rieoei.org/deloslectores/1101Gonzalez.pdf.

Fromm. L. y Ramos, V. (2009). La Práctica pedagógica cotidiana: hacia Nuevos modelos de investigación en el aula. San José, C.R.: Coordinación Educativa y Cultural Centroamericana.

Hernández, R; Fernández, C; y Baptista, P. (2006) Metodología de la Investigación. México. Editorial Ultra. Cuarta edición.

Hidalgo, J. \& Valverde, M. (2007). Influencia de las técnicas didácticas en la motivación que tengan los estudiantes hacia las clases de física de quinto año en dos colegios públicos costarricenses. Costa Rica: Heredia. Universidad Nacional.

Pozo, J., \& Gómez, M. (1998). Aprender y enseñar ciencia: del conocimiento cotidiano al cientifico. España: Madrid: EDICIONES MORATA, S.L. 
Restrepo, B. (s.f). Una variante pedagógica en la investigación-acción educativa. Revista Iberoamericana de Educación. Recuperado de http://www.rieoei.org/deloslectores/370Restrepo.PDF.

Rojas, R. (2006). La Creación de Zonas de Desarrollo Próximo como estrategia de la enseñanza aprendizaje constructivista en la resolución de problemas lógicomatemáticos, en una clase de física del ciclo de educación diversificada de la institución pública costarricense. Costa Rica: Heredia. Universidad Nacional.

Santamaría, M. (2006). ¿Cómo evaluar los aprendizajes en el aula? Costa Rica: EUNED

Sandonís, R.(s.f).Introducción: Física y lenguaje, las matemáticas. Recuperado de http:// ocw.unican.es/ensenanzas-tecnicas/fundamentos-fisicos-de-la-ingenieria/ material-de-clase 